\title{
Risk Factors and Intervention Status of Cardiovascular Disease in Elderly Patients with Coronary Heart Disease
}

\author{
Bin Deng1, Min Guo ${ }^{2 *}$ \\ ${ }^{1}$ The Fourth People's Hospital of Haikou, Haikou, China \\ ${ }^{2}$ Hainan General Hospital, Haikou, China \\ Email: *g2002m@163
}

How to cite this paper: Deng, B. and Guo, M. (2020) Risk Factors and Intervention Status of Cardiovascular Disease in Elderly Patients with Coronary Heart Disease. Health, 12, 857-865.

https://doi.org/10.4236/health.2020.127063

Received: June 21, 2020

Accepted: July 24, 2020

Published: July 27, 2020

Copyright $\odot 2020$ by author(s) and Scientific Research Publishing Inc. This work is licensed under the Creative Commons Attribution International License (CC BY 4.0).

http://creativecommons.org/licenses/by/4.0/

\begin{abstract}
To analyze the correlation between coronary heart disease and the possible risk factors such as age, gender, hypertension, dyslipidemia, smoking, diabetes, uric acid and plasma fibrinogen, to screen the main risk factors of coronary heart disease in the elderly and evaluate the intervention status of coronary heart disease in the elderly. In the unchangeable risk factors, age is still the main risk factor of coronary heart disease; in terms of gender, the onset age of female coronary heart disease is later than that of male, but the complications and mortality rate are higher than that of male, and the risk factors are more than that of male. The first level prevention needs doctors and patients to establish a mutual trust relationship, and actively intervene to reduce the total risk of cardiovascular disease. Drug compliance management should be strengthened in secondary prevention.
\end{abstract}

\section{Keywords}

Coronary Heart Disease, Cardiovascular, Risk Factors, Intervention Status

\section{Background}

Coronary heart disease (CHD) is the most common disease in the elderly, and it is the primary cause of death for people over 65 years old. Primary and secondary prevention of coronary heart disease is an indisputable public health problem in the world. The direct cause of atherosclerosis has not been studied. Chen haozhu et al. [1] classified risk factors into four categories: 1) Risk factors: age, gender, family history and gene; 2) Risk factors that can be adjusted: smoking, atherogenic diet, drinking and physical exercise; 3) Metabolic risk factors: dysli- 
pidemia, hypertension, obesity, diabetes mellitus, metabolic syndrome; 4) Disease markers: calcium plaque, inflammation, interventional effect, load test results, left ventricular hypertrophy, history of cardiovascular disease (myocardial infarction, stroke, angina peripheral vascular disease). A large number of studies have shown that the disease is directly or indirectly related to a variety of risk factors. Effective preventive measures are taken for the risk factors with clear causal relationship with cardiovascular disease, with clear effect. Active implementation of intervention measures can reduce the economic burden of patients and improve the quality of life.

\section{The Relationship between the Risk Factors of Coronary Heart Disease and Coronary Heart Disease}

\subsection{Age and Coronary Heart Disease}

With the increase of age, the prevalence of coronary heart disease increased significantly, especially the intima of artery wall proliferated with the increase of age, and the risk factors such as hypertension, diabetes and dyslipidemia also increased with the increase of age [2]. With the increase of cardiovascular disease incidence, the incidence of cardiovascular surgery has increased dramatically. The incidence rate of arrhythmia in elderly patients is higher. Elderly patients receiving vascular surgery have higher risk of vascular events than young patients [3]. With the increase of age, the prevalence of coronary heart disease increased significantly, especially the intima of artery wall increased with age, such as hypertension, diabetes and dyslipidemia. With the increase of cardiovascular disease incidence, the incidence of cardiovascular surgery has increased dramatically. The incidence rate of arrhythmia in elderly patients is higher. Elderly patients receiving vascular surgery have higher risk of vascular events than young patients. Fu Jun's research findings [4], the age of coronary heart disease group was higher than that of non-coronary heart disease group, and the prevalence rate of old myocardial infarction was more than 85 years old group. Multivariate logistic regression analysis showed that age was an independent risk factor of coronary heart disease; age was an unchangeable risk factor, and it was also a risk factor, so there were no effective intervention measures.

\subsection{Gender and Coronary Heart Disease}

Most of the data show that the incidence of coronary heart disease in postmenopausal women is significantly increased. It is considered that the lack of estrogen is the main factor in the incidence of coronary heart disease in women, and estrogen has the effect of anti-atherosclerosis. Therefore, it is necessary to explore the effect of estrogen replacement therapy on the incidence of coronary heart disease in postmenopausal women, which is an effective intervention measure, but there is potential to increase tumor risk, so application should be cautious. The risk factors of cardiovascular disease are younger and more common in male patients. Lifestyle change can reduce most of the risk factors of cardiovascular disease [4]. 


\subsection{Hypertension and Coronary Heart Disease}

The prevalence of coronary heart disease in patients with hypertension compared with patients without hypertension of the same age, there is a dose relationship between hypertension and the degree of coronary heart disease. The higher the blood pressure level, the more severe the degree of coronary artery stenosis. The incidence rate of coronary heart disease and stroke in hypertensive patients is significantly higher than that in normotensive patients. Studies have found that pulse pressure can be used as a risk factor for stroke in middle-aged and elderly hypertensive patients when systolic blood pressure is normal [5]. Systolic blood pressure increases with age, becoming a strong predictor of cardiovascular events (especially for women). Signs of human cardiovascular aging include increased systolic blood pressure, increased pulse pressure, increased pulse rate and decreased left ventricular ejection fraction [6]. According to data from the Framingham Heart Study, 90\% of people with normal blood pressure at the age of 55 may already have high blood pressure [7]. It was found that there was a linear relationship between the rise of blood pressure and the risk of cardiovascular disease. For people over 60 years old, within the range of 115/75 $\mathrm{mmHg}$ to $185 / 115 \mathrm{mmhg}$, the risk of cardiovascular disease doubled with every $20 \mathrm{mmHg}$ increase of systolic pressure or $10 \mathrm{mmHg}$ increase of diastolic pressure, and the risk and mortality of coronary heart disease increased with postural hypotension [8]. The goal of hypotensive treatment is to gradually reduce blood pressure to normal or close to normal level, without insufficient blood supply due to excessive hypotension in the heart, brain, kidney and other major organs. At this time, it is the appropriate hypotensive level, which can be maintained by long-term medication.

\subsection{Dyslipidemia and Coronary Heart Disease}

At present, it has been found that the abnormal lipid metabolism is closely related to atherosclerosis. Lipid is the general term of lipids in plasma, mainly composed of triglycerides, phospholipids, cholesterol and its esters, and free fatty acids. There are many theories about the pathogenesis of coronary heart disease, the first one is the theory of fat infiltration. The level of blood lipid plays an important role in the occurrence and development of atherosclerosis. Studies have found that high systolic blood pressure and low HDL-C (High-Density Lipoprotein HDL-C) can increase the incidence rate of cardiovascular events [9]. Triglyceride and HDL-C are independent predictors of coronary heart disease mortality in middle-aged and elderly women. The combination of high triglyceride and low HDL-C can double the prevalence of coronary heart disease in middle-aged and elderly women, and also increase the risk of coronary heart disease mortality [10]. A large number of animal experiments, genetic and epidemiological data have confirmed that cholesterol level has a great correlation with coronary heart disease, arteriosclerosis, There is no doubt that lowering hyperlipidemia can reduce the risk of coronary heart disease. 


\subsection{Smoking and Coronary Heart Disease}

Most studies have found that nicotine can stimulate sympathetic nerves to cause arterioles to constrict, increase blood pressure, reduce beneficial HDL, increase harmful LDL, and promote the formation of arteriosclerosis; The incidence rate of insulin resistance was 20, or more cigarettes per day. The risk of myocardial infarction was three times higher than that of non-smokers, former smokers and smokers or smokers. Smoking can lead to the increase of blood pressure, heart rate, platelet activation and coronary plaque instability. The risk of coronary heart disease increases linearly with the number of cigarettes per day. Long-term smoking can accelerate the process of atherosclerosis and affect endotheliumdependent coronary artery relaxation. Chinese studies have shown that smoking is independently related to the degree of coronary atherosclerosis, and there is a linear dose-response relationship between them [11]. Therefore, smoking cessation should be one of the main preventive measures for coronary heart disease, thereby reducing the incidence rate and mortality of coronary heart disease.

\subsection{Diabetes and Coronary Heart Disease}

In 1999, the American Heart Association (AHA) proposed that "diabetes is a cardiovascular disease"; diabetes is an independent predictor of obstructive coronary disease [12]. Japanese researchers found that the prevalence of abdominal obesity and insulin resistance increased with age [13]; Insulin resistance can also activate the coagulation system, inhibit the fibrinolysis system and cause thrombosis; insulin can reduce the expression of inflammatory factors [14]. Impaired fasting glucose regulation is a part of the metabolic syndrome and an important risk factor of atherosclerosis. Most patients with acute coronary syndrome have abnormal glucose metabolism, which is also common in patients with stable angina pectoris. Only obesity is an independent predictor of coronary atherosclerosis and abnormal glucose metabolism [15]. In elderly women, the level of estrogen in the body is reduced, and endocrine regulation disorder is common, which is easy to lead to glucose metabolism disorder [16].

\subsection{Plasma Fibrinogen and Coronary Heart Disease}

Who proposed in 1999 that the increase of plasma fibrinogen is also a risk factor of cardiovascular disease [17]. Plasma fibrinogen level is closely related to the occurrence, development and outcome of coronary heart disease. Fibrinogen and traditional cardiovascular risk factors are used as biomarkers of cardiovascular disease in high-risk population [18]. The level of plasma fibrinogen in coronary heart disease group was higher than that in non-coronary heart disease group. The level of plasma fibrinogen in patients with unstable angina and old myocardial infarction was higher than other patients, but there was no statistical difference. Clinical data show that most of the patients with elevated fibrinogen level may have myocardial infarction or sudden death, and the higher the level of fibrinogen, the greater the risk; there is no active and effective intervention on 
plasma fibrinogen in clinical, the main reason is that there are many basic diseases and many kinds of drugs in elderly patients with coronary heart disease, and large-scale clinical research is needed to confirm the plasma fibrinogen The clinical effect of the intervention and control of tretinogen.

\subsection{Blood Uric Acid and Coronary Heart Disease}

Uric acid is the end product of purine nucleotide metabolism in human body. It is mainly decomposed from nucleic acid or purine in food, and finally discharged from kidney. The production of uric acid is related to glycolysis, which can cause fat metabolism disorder. As an independent risk factor of cardiovascular disease, blood uric acid is still controversial [19]. The measurement of serum uric acid level can not improve the risk prediction of coronary heart disease. The detection of this index has not been widely used in the general population [20]. Uric acid level may be a useful biomarker for risk stratification in patients with ST segment elevation myocardial infarction [21], and it can also predict type 2 diabetes [22]. Uric acid is an independent risk factor for all-cause death and coronary heart disease death in women, but it is not an independent risk factor in men.

\section{Intervention Status of Risk Factors}

According to the research data, aspirin, B-receptor blocker, lipid-lowering drugs and $\mathrm{ACE}$ inhibitors are effective in secondary prevention of coronary heart disease in individuals over 65 years old, and their efficacy is better than that in young patients. These drugs should be prescribed for all elderly patients without contraindications [23]. Ongoing research and future clinical trials will shed more light on the benefits of secondary prevention of coronary heart disease, especially in patients older than 75 years [24]; aspirin, ACE inhibitors, B-receptor blockers and statins reduce the risk of long-term mortality, especially in the elderly who may benefit from statins [25]. In patients with cardiovascular risk factors but not diagnosed with cardiovascular disease, statins can significantly improve survival and reduce the risk of major cardiovascular events [26] in clinical practice, poor vascular drug compliance is a common and common phenomenon, and drug compliance should be the goal of improving intervention in patients with coronary heart disease [27]. In stable coronary heart disease patients, the evidence of selective percutaneous coronary intervention (PCI) is generally insufficient. Future research should focus on providing more sufficient clinical evidence for medical decision-making [28]. Among the changeable risk factors, smoking was the best intervention effect, followed by blood lipid, and the worst intervention was fasting blood glucose; The control rate of blood pressure in elderly patients with hypertension is still low, and it is easy to be affected by body position change; uric acid and plasma fibrinogen are new risk factors which are not intervened. In the unchangeable risk factors, age is still the main risk factor for coronary heart disease; in terms of gender, the onset age of 
coronary heart disease in women is later than that in men, but the complications and mortality are higher than men, and the risk factors are more than men. In primary prevention, doctors and patients should establish mutual trust relationship, actively and effectively implement intervention to reduce the total risk of cardiovascular disease; in secondary prevention, drug compliance management should be strengthened.

1) among the changeable risk factors, smoking was the best one, blood lipid was the second, fasting blood glucose was the worst one; the blood pressure of the elderly patients with hypertension fluctuated greatly, and they were easily affected by the change of body position; the new risk factors of uric acid and plasma fibrinogen were not intervened. In the unchangeable risk factors, age is still the main risk factor of coronary heart disease; in terms of gender, the onset age of female coronary heart disease is later than that of male, but the complications and mortality rate are higher than that of male, and the risk factors are more than that of male. The same patient often has multiple risk factors at the same time, the evolution of coronary heart disease rarely depends on a single risk factor, the comprehensive harm of multiple risk factors far exceeds the role of a single risk factor.

2) Most of the elderly patients with coronary heart disease have many basic diseases, complex condition, many kinds of drugs and poor treatment effect. In clinical practice, we should pay attention to the comprehensive evaluation of risk factors of coronary heart disease. For patients with a history of early coronary heart disease (male before 55 years old, female before 65 years old, coronary heart disease), we should actively improve their lifestyle, quit smoking and limit alcohol, participate in physical exercise regularly, and control blood Fat, blood pressure and blood sugar are at appropriate levels; The metabolic syndrome related to insulin resistance should be found in time, because it increases the risk of coronary heart disease. It is an essential link in the treatment of cardiovascular disease to deal with the lipid abnormality of patients with coronary heart disease or those with risk factors of early coronary heart disease. For diabetics, hypercholesterolemia needs active treatment and effective and feasible control. Patients with coronary heart disease should be prevented from myocardial infarction, stroke and peripheral vascular disease; patients without coronary heart disease should be classified into diabetic patients, high-risk non-diabetic patients and low-risk non-diabetic patients. Although the age adjusted risk rate is reduced, more secondary prevention measures should be taken, which is the key to the prevention and treatment of cardiovascular diseases.

To sum up, with the further understanding of atherosclerotic pathophysiology, lifestyle, biochemical factors and genes related to coronary heart disease, no matter the risk factors of atherosclerosis are acquired behavior (such as smoking), genetic quality (such as familial hyperlipidemia) or laboratory test index (cholesterol or C-reactive protein), age-adjusted cardiovascular system The death rate of the disease has been greatly reduced. Although most of the studies 
focus on the change of single risk factors, in theory, the risk of atherosclerosis is multiplied by the cross influence, mutual cause and effect and synergy of multiple risk factors, and the future situation of atherosclerosis prevention is still grim. It is still difficult to change some risk factors. The key to determine whether the intervention is successful or not is doctors. In terms of cost/benefit ratio and risk/benefit ratio, further research is needed to strive for maximum social benefits.

\section{Foundation Project}

Supported by Hainan Natural Science Foundation (Project No. 817393).

\section{Conflicts of Interest}

The authors declare no conflicts of interest regarding the publication of this paper.

\section{References}

[1] Lynn, M.M. (2000) Primary and Secondary Prevention of Coronary Heart Disease. Evidence Offers Multiple Strategies. Advance for Nurse Practitioners, 8, 36-41.

[2] Hoeks, S.E., Reimer, W.J., Schouten, O., et al. (2008) Statin Use in the Elderly: Results from a Peripheral Vascular Survey in the Netherlands. Journal of Vascular Surgery, 48, 891-895. https://doi.org/10.1016/j.jvs.2008.04.073

[3] Andersen, K.K., Andersen, Z.J. and Olsen, T.S. (2010) Age- and Gender-Specific Prevalence of Cardiovascular Risk Factors in 40102 Patients with First-Ever Ischemic Stroke: A Nmionwide Danish Study. Stroke, 41, 2768-2774. https://doi.org/10.1161/STROKEAHA.110.595785

[4] Fu, J. (2012) Risk Factors Analysis and Intervention Status of Cardiovascular Disease in Elderly Patients with Coronary Heart Disease. Hebei Medical University, Shijiazhuang.

[5] Okada, K., Iso, H., Cui, R., Inoue, M. and Tsugane, S. (2011) Pulse Pressure Is an Independent Risk Factor for Stroke among Middle-Aged Japanese with Normal Systolic Blood Pressure: The JPHC Study. Journal of Hypertension, 29, 319-324. https://doi.org/10.1097/HJH.0b013e32834143d9

[6] Kass, D. (2002) Age-Related Changes in Ventricular-Arterial Coupling Pathophysiologic Implications. Heart Failure Reviews, 7, 51-62.

[7] Vasan, R.S., Beiser, A., Seshadrl, S., et al. (2002) Residual Lifetime Risk for Developing Hypertension in Middle-Aged Women and Man: The Framingham Heart Study. JAMA, 287, 1003-1010. https://doi.org/10.1001/jama.287.8.1003

[8] Verwoert, G.C., Mattace-Raso, F.U.S., Hofrnan, A., et al. (2008) Orthostatic Hypotension and Risk of Cardiovascular Disease in Elderly People: The Rotterdam Study. Journal of the American Geriatrics Society, 56, 1816-1820. https://doi.org/10.1111/j.1532-5415.2008.01946.x

[9] De Freitas, E.V., Brandao, A.A., Pozzan, R., et al. (2011) Importance of High-Density Lipoprotein-Cholesterol (HDL-C) Levels to the Incidence of Cardiovascular Disease (CVD) in the Elderly. Archives of Gerontology and Geriatrics, 52, 217-222. https://doi.org/10.1016/j.archger.2010.03.022

[10] Mazza, A., Tikhonoff, V., Schiavon, L. and Casiglia, E. (2005) Triglycerides + 
High-Density-Lipoprotein-Cholesterol Dyslipidaemia, a Coronary Risk Factor in Elderly Women: The Cardiovascular Study in the Elderly. Internal Medicine Journal, 35, 604-610. https://doi.org/10.1111/j.1445-5994.2005.00940.x

[11] Luan, Y.X. (2010) Detection and Correlation Analysis of Serum h-CRP and IMT in 106 Elderly Patients with Coronary Heart Disease. Shandong Medicine, 50, 61-62.

[12] Veeranna, V., Pradhan, J., Niraj, A., et al. (2010) Traditional Cardio-Vascular Risk Factors and Severity of Angiographic Coronary Artery Disease in the Elderly. Preventive Cardiology, 13, 135-140.

[13] Sakurai, T., Iimuro, S., Araki, A., Umegaki, H., Ohashi, Y., Yokono, K. and Ito, H. (2010) Age-Associated Increase in Abdominal Obesity and Insulin Resistance, and Usefulness of AHA/NHLBI Definition of Metabolic Syndrome for Predicting Cardiovascular Disease in Japanese Elderly with Type 2 Diabetes Mellitus. Gerontology, 56, 141-149. https://doi.org/10.1159/000246970

[14] Paresh, D., Ajay, C., Priya, M.E. and Husam, G. (2007) Anti-Inflammatory Effects of Insulin. Current Opinion in Clinical Nutrition and Metabolic Care, 10, 511-517. https://doi.org/10.1097/MCO.0b013e3281e38774

[15] Lodarczyk, A. and Strojek, K. (2008) Glucose Intolerance, Insulin Resistance and Metabolic Syndrome in Patients with Stable Angina Pectoris. Obesity Predicts Coronary Atherosclerosis and Dysglycemia. Polish Archives of Internal Medicine, 118, 719-726. https://doi.org/10.20452/pamw.538

[16] Glueck, C.J., Morrison, J.A., Goldenberg, N. and Wang, P. (2009) Coronary Heart Disease Risk Factors in Adult Premenopausal White Women with Polycystic Ovary Syndrome Compared with a Healthy Female Population. Metabolism, 58, 714-721. https://doi.org/10.1016/j.metabol.2009.02.005

[17] HOPE/HOPE-TOO Study Investigators (2005) Long-Term Effects of Ramipril on Cardiovascular Events and on Diabetes: Results of the Hope Study Extension. Circulation, 112, 1339-1346. https://doi.org/10.1161/CIRCULATIONAHA.105.548461

[18] Maple-Brown, L.J., Cunninhan, J., Nandi, N., Hodge, A. and O’Dea, K. (2010) Fibrinogen and Associated Risk Factors in a High-Risk Population: Urban Indigenous Australians, the DRUID Study. Cardiovascular Diabetology, 9, Article No. 69. https://doi.org/10.1186/1475-2840-9-69

[19] Strasak, A., Ruttmann, E., Brant, L., et al. (2008) Serum Uric Acid and Risk of Cardiovascular Mortality: A Prospective Long-Term Study of 83, 683 Austrian Men. Clinical Chemistry, 54, 273-284. https://doi.org/10.1373/clinchem.2007.094425

[20] Wheeler, J.G., Juzwishin, K.D.M., Eiriksdottir, G., Gudnason, V. and Danesh, J. (2005) Serum Uric Acid and Coronary Heart Disease in 9,458 Incident Cases and 155,084 Controls: Prospective Study and Meta-Analysis. PLoS Medicine, 2, e76. https://doi.org/10.1371/journal.pmed.0020076

[21] Akpek, M., Kaya, M.G., Uyarel, H., et al. (2011) The Association of Serum Uric Acid Levels on Coronary Flow in Patients with STEMI Undergoing Primary PCI. Atherosclerosis, 219, 334-341. https://doi.org/10.1016/j.atherosclerosis.2011.07.021

[22] Onat, A., Uyarel, H., Hergenc, G., et al. (2006) Serum Uric Acid Is a Determinant of Metabolic Syndrome in a Population-Based Study. American Journal of Hypertension, 19, 1055-1062. https://doi.org/10.1016/j.amjhyper.2006.02.014

[23] John, G.C., Catarina, I.K., William, J.R., et al. (2011) Number of Coronary Heart Disease Risk Factors and Mortality in Patients with First Myocardial Infarction. $J A M A, 306,2120-2127$. https://doi.org/10.1001/jama.2011.1654

[24] Dombrook-Lavender, K.A., Roth M.T. and Pieper, J.A. (2003) Secondary Prevention of Coronary Heart Disease in the Elderly. Annals of Pharmacotherapy, 37, 1867-1876. 
https://doi.org/10.1345/aph.1D026

[25] Feringa, H.H.H., Bax, J.J., Karagianis, S.E., et al. (2009) Elderly Patients Undergoing Major Vascular Surgery: Risk Factors and Medication Associated with Risk Reduction. Archives of Gerontology and Geriatrics, 48, 116-120.

https://doi.org/10.1016/j.archger.2007.11.003

[26] Brugts, J.J., Yetgin, T., Hocks, S.E., et al. (2009) The Benefits of Statins in People without Established Cardiovascular Disease but with Cardiovascular Risk Factor: Meta-Analysis of Randomised Controlled Trials. BMJ, 338, b2376.

https://doi.org/10.1136/bmj.b2376

[27] Ho, P.M., Magid, D.M., Shetterly, S.M., et al. (2008) Medication Non-Adherence Is Associated with a Broad Range of Adverse Outcomes in Patients with Coronary Artery Disease. American Heart Journal, 155, 772-779.

https://doi.org/10.1016/j.ahj.2007.12.011

[28] Lin, G.A., Dudley, R.A. and Redberg, R.F. (2007) Cardiologists' Use of Percutaneous Coronary Interventions for Stable Coronary Artery Disease. Archives of Internal Medicine, 167, 1604-1609. https://doi.org/10.1001/archinte.167.15.1604 